\title{
LA RÉGULATION DES PROFESSIONS DE SANTÉ EN FRANCE*
}

\author{
Health professions regulation in France
}

${ }^{1}$ Université Paris Descartes. Nantes, France.

${ }^{2}$ Université Paris Descartes. Paris, France.

Correspondance: stephane.brissy@univ-nantes.fr

Reçu: 19/06/2018. Révisé: 19/09/2018. Approuvé: 24/09/2018.

\footnotetext{
*Article original développé dans le cadre de la recherche "Arrangements institutionnels pour la médiation des différents établissements de santé des professions de la santé au Brésil: itinéraire judiciaire et administratif pour la création de nouvelles professions de la santé et vision du Bureau de réglementation des travaux de santé / Ministère de la santé brésilien", réalisée par le Centre d'études et de recherche en droit de la santé (Cepedisa) en 2018.
} 


\section{RÉSUMÉ}

La régulation juridique des professions de santé s'inscrit dans une politique de santé publique et participe de la mise en place d'un système de santé de qualité accessible à tous. Elle a cependant été longtemps accaparée par des corps professionnels, essentiellement le corps médical, défendant un système libéral et une autonomie juridique des professions. Lorganisation professionnelle de la santé s'en ressent encore aujourd'hui mais tend de plus en plus à devenir l'affaire de tous les citoyens.

\section{Mots-clés}

Agences Régionales de Santé; Déontologie; Compétences; Convention d’Exercise Professionel; Pouvoir Normatif.

\section{ABSTRACT}

The legal regulation of health professions is part of a public health policy and contributes to the establishment of a quality health system accessible to all. However, it has long been monopolized by professional bodies, mainly the medical profession, defending a liberal system and a legal autonomy of professions. The legal regulation of health still suffers today but tends more and more to become the business of all citizens.

\section{Keywords}

Deontology; Normative Power; Professional Practice Agreement; Regional Healthcare Agencies; Scopes. 


\section{Introduction}

Lactuelle régulation des professions de santé en France est le fruit d'un processus long et mouvementé. L'encadrement juridique de l'activité des professionnels de santé dont il sera question ici ne peut être analysé sans avoir conscience des oppositions et des coalitions sociales et politiques qui ont entouré et entourent encore l'édification du système de protection sociale et du système de santé.

Si la loi affirme aujourd'hui que «la politique de santé relève de la responsabilité de l'Etat» ${ }^{1}$, il n'en a pas toujours ainsi. La construction d'un système de protection sociale n’a été possible quavec la participation des organisations professionnelles de travailleurs et d’employeurs, pour donner corps à l'idée de démocratie sociale $^{2}$. Parallèlement, le modèle d'une organisation sanitaire calquée sur un exercice libéral et individuel de la médecine affranchi de l'autorité de l'Etat a imprégné le fonctionnement du système de santé en France ${ }^{3}$. Cette conception de l'activité professionnelle en santé impliquait une revendication forte dautorégulation et dès lors d'importants pouvoirs normatifs confiés aux représentants de chaque profession, par la voie des organisations syndicales mais aussi d'un ordre professionnel ayant vocation à réguler l'ensemble d'une profession. Sans bénéficier d'une capacité totale d'autorégulation, le corps médical a toutefois constitué pendant longtemps le centre de l'organisation sanitaire en France et la régulation des professions de santé, médicales et non médicales en a subi les conséquences.

Au prix de quelques scandales sanitaires mettant en évidence les dangers d'un manque d'intervention de l'Etat dans la santé, les pouvoirs publics se sont saisis davantage des questions de santé et se sont attelés à la construction d'une politique nationale de santé tendant à dépasser les corps professionnels. Mais la régulation des professions de santé nest pas pour autant le fait de l'Etat seul. Non seulement l'héritage historique de la santé en France ná pas été totalement gommé mais qui plus est, les processus de régulation sont globalement de plus en plus partagés entre les pouvoirs publics et d’autres acteurs disposant d'un pouvoir normatif ou d'un «quasi» pouvoir normatif. Dans le même temps, l'Etat français se charge de décliner, à travers une stratégie annuelle ou pluriannuelle de santé, les objectifs auxquels doit répondre le système de santé et qui sont

[...] la promotion de conditions de vie favorables à la santé, l'amélioration de létat de santé de la population, la réduction des inégalités sociales et territoriales, l'égalité entre les femmes et les

\footnotetext{
${ }^{1}$ Art. L 1411-1 alinéa 2 du Code de la santé publique (CSP). LEGIFRANCE. Disponible en: <https://www. legifrance.gouv.fr/affichCode.do?cidTexte=LEGITEXT000006072665>. Accès en: 19 Sept. 2018.

${ }^{2}$ BEC, C. La sécurité sociale: une institution de la démocratie. Paris: Gallimard, 2014.

${ }^{3}$ HASSENTEUFEL, P. Les médecins face à l'Etat: une comparaison européenne. Paris: Presses de Sciences Po., 1997; TABUTEAU, D. L'avenir de la médecine libérale et le spectre de Monsieur Bovary. Droit Social, Paris, n. 4, p. 383, 2009.

${ }^{4}$ COMMAILLE, J.; JOBERT, B. (Dir.). Les métamorphoses de la régulation politique. Paris: LGDJ, 1998. (coll. Droit et société).
} 
hommes et [...] la meilleure sécurité sanitaire possible et l'accès effectif de la population à la prévention et aux soins ${ }^{5}$.

S’y ajoute la maîtrise des dépenses de santé.

Le partage des pouvoirs de régulation, qui peut amener à parler de gouvernance plus que de gouvernement du système de santé, n’enlève pas à l'Etat la responsabilité d’atteindre ces objectifs centrés sur l'amélioration de la santé de tous. Les parcelles d'autonomie juridique reconnues aux professionnels de santé ne peuvent dès lors conduire à une «autorégulation» des professions de santé (I). Et c'est parce qu'ils sont essentiels que ces objectifs doivent être intégrés dans la régulation des compétences professionnelles (II) et des libertés d’offres et de tarifs des professionnels (III).

\section{Une autonomie sans autoregulation}

L' Etat français a repris en main l'organisation de la politique de santé pour que soient au mieux assurés ses objectifs et il serait très excessif de parler aujourd'hui d'une véritable autorégulation des professions de santé. Certaines d'entre elles disposent néanmoins d'une autonomie juridique qui passe par plusieurs canaux dont la multiplicité s'explique à la fois par la différence de rôles attribués aux représentants et par le fait qu'un professionnel de santé peut exercer son activité de différentes manières : soit en tant que professionnel libéral, c'est-à-dire pour son propre compte et selon une organisation du travail qu'il détermine ; soit pour le compte d'un établissement, public ou privé, suivant une organisation du travail qu'il n’a pas décidée. Sans occulter les nombreuses formes de représentation professionnelle, arrêtons-nous dans un premier temps sur les deux principales catégories de représentants disposant d'un pouvoir normatif, à savoir les ordres professionnels et les syndicats.

\section{Les ordres professionnels}

Le Code de la santé publique prévoit l'existence d'un ordre professionnel et d'un code de déontologie pour sept des professions qu'il identifie comme professions de santé : médecins, sages-femmes, chirurgiens-dentistes, infirmiers, masseurs-kinésithérapeutes, pharmaciens et pédicures-podologues. Pour les autres, les règles légales communes s'appliquent ainsi que des dispositions spécifiques qui concernent en grande partie le droit d'exercer la profession et les actes ou activités pouvant être accomplis par les professionnels habilités.

Le rôle général des ordres professionnels va au-delà de la seule représentation des intérêts des professionnels ${ }^{6}$. L'ordre et les règles déontologiques doivent assurer

${ }^{5}$ Art. L 1411-1, alinéa 3 du CSP.

${ }^{6}$ BRISSY, S. La régulation des professions de santé par les ordres professionnels en droit français et européen. Revista de Direito Sanitário, São Paulo, v. 17, n. 3, p. 77, fev. 2017. Disponible en: <http://www.revistas.usp. br/rdisan/article/view/127777/124774>. https://doi.org/10.11606/issn.2316-9044.v17i3p77-106. 
la reconnaissance sociale de la profession et sa cohésion au regard de valeurs qui dépassent les seuls intérêts de ses membres. C'est pour cette raison que les codes de déontologie présents dans le Code de la santé publique affirment tous que les professionnels concernés sont au service des individus et de la santé publique, qu'ils doivent agir dans le respect de la personne et de sa dignité et que l'ordre doit notamment veiller à maintenir des principes de moralité, de probité, de compétence, de dévouement, à préserver l'indépendance des professionnels et à promouvoir la santé publique et la qualité des soins.

L' existence d'un ordre professionnel et de règles déontologiques propres se justifient par la reconnaissance de compétences spécifiques et, globalement, d'une profession dont l'utilité sociale est ainsi affirmée. Mais dans le même temps, cette fonction sociale des professions de santé souligne que les principes déontologiques qui la protègent ne concernent pas seulement les relations entre professionnels euxmêmes et justifie qu'ils ne soient pas uniquement fixés par eux. Pour qu'un code de déontologie relatif à une profession de santé acquière toute sa valeur juridique, il faut ainsi qu'il soit approuvé par un décret pris après avis du Conseil d'Etat, même si son contenu est en toute ou partie élaboré initialement par lordre professionnel.

Le pouvoir disciplinaire des ordres leur permettant de sanctionner un professionnel qui ne respecte pas une ou des règles déontologiques est également limité par l'intervention de l'Etat. L'organisation des juridictions disciplinaires et les peines quelles peuvent prononcer sont fixées par des règles étatiques. Quant aux décisions rendues par les instances ordinales, elles peuvent être contestées devant le Conseil d'Etat.

Les règles déontologiques et leur application par les ordres contribuent ainsi à l'existence d'un ordre juridique professionnel intégré à lordre juridique étatique?

Sans être indépendant du droit et des institutions de l'Etat, cet ordre juridique professionnel n'en manifeste pas moins la reconnaissance aux professions d'une capacité de régulation, et dès lors d'une certaine autonomie juridique. Les instances d'un ordre professionnel disposent ainsi du pouvoir de prendre des décisions qui s'imposent aux professionnels sans le secours des pouvoirs publics.

Une procédure disciplinaire engagée contre un professionnel de santé devant lordre est indépendante d'une procédure engagée, contre le même professionnel et pour les mêmes faits, devant le juge civil ou le juge pénal. Par exemple, le fait qu'un patient se soit désisté de l'action intentée contre un professionnel devant la justice civile ne prive pas d'objet le litige introduit devant la juridiction disciplinaire de lordre ${ }^{8}$. La saisine des instances disciplinaires de lordre est par ailleurs ouverte

${ }^{7}$ BAILLY, J. M. Les déontologies. Paris: Presses universitaires d'Aix-Marseille, 2001. p. 408 et s.

${ }^{8}$ Conseil d'Etat (CE), 28 mars 2018, $n{ }^{\circ}$ 405077. LEGIFRANCE. Disponible en: <https://www.legifrance.gouv.fr/ affichJuriAdmin.do?oldAction=rechJuriAdmin\&idTexte=CETATEXTO00036753597>. Accès en: 19 Sept. 2018. 
à d'autres personnes que les seuls professionnels ou patients. Le Conseil d'Etat a notamment précisé récemment qu'un employeur peut saisir la chambre disciplinaire de l'ordre des médecins lorsqu'il estime qu'un certificat dans lequel un médecin du travail fait état d'un lien entre la santé d'un salarié et ses conditions de travail sans avoir vérifié personnellement ces conditions est un certificat tendancieux, en contradiction avec les obligations déontologiques qui s'imposent à tout médecin ${ }^{9}$. Le droit de saisir les instances disciplinaires est ainsi largement ouvert.

Le pouvoir disciplinaire de l'ordre cesse toutefois lorsqu'une juridiction disciplinaire spécifique est chargée de statuer sur les poursuites disciplinaires, ce qui est le cas par exemple concernant les professeurs ou maître de conférences des universités qui sont également praticiens hospitaliers, pour des faits qui ne sont pas détachables de leur activité d'enseignant-chercheur ${ }^{10}$.

L' application des principes déontologiques par l'ordre peut aussi passer par une délibération générale, dont le Conseil d’Etat a considéré quelle présente un caractère réglementaire, compte tenu des missions de l'ordre pour l'application des règles déontologiques si la délibération est formulée de manière impérative ${ }^{11}$.

En complément de la régulation par les ordres, l'organisation d'une profession de santé emprunte aussi la voie de la négociation collective à laquelle les organisations syndicales sont parties.

\section{Les organisations syndicales et la voie conventionnelle}

La variété des modes d'exercice des professions de santé implique une certaine diversité dans le rôle normatif joué par les syndicats. Celui-ci s'exprime parfois par des consultations obligatoires mais surtout par une capacité juridique à négocier et adopter collectivement des textes qui auront une valeur normative.

Concernant les professionnels exerçant dans les hôpitaux publics et ayant la qualité de fonctionnaire hospitalier, les organisations syndicales de fonctionnaires participent au niveau national à des négociations avec les représentants du

\footnotetext{
${ }^{9} \mathrm{CE}, 6$ juin 2018, ${ }^{\circ}$ 405453: "la mention, dans un certificat médical produit par un salarié devant le juge prud'homal dans le cadre d'un litige l'opposant à son employeur, d'un 'enchaînement délétère de pratiques maltraitantes' de la part de ce dernier, lésait cet employeur de manière suffisamment directe et certaine pour que sa plainte dirigée contre le médecin auteur de ce certificat soit recevable". LEGIFRANCE. Disponible en: <https://www.legifrance.gouv.fr/affichJuriAdmin.do?oldAction=rechJuriAdmin\&idTexte=CETATEXTO000 37022279\&fastReqld=1018602938\&fastPos=1>. Accès en: 19 Sept. 2018.

${ }^{10} \mathrm{CE}, 13$ avr. $2018, n^{\circ} 406887$ : professeur des universités-praticien hospitalier poursuivi pour avoir diffusé à des étudiants un sujet d'examen polémique. JURICAF. Disponible en: <https://juricaf.org/arret/FRANCECONSEILDETAT-20180413-406887>. Accès en: 19 Sept. 2018.

${ }^{11} \mathrm{CE} 17$ nov. 2010, $\mathrm{n}^{\circ} 332771$ : délibération du Conseil national de l’ordre des médecins préconisant aux médecins de ne pas collaborer avec les ostéopathes au sein des maisons de santé. LEGIFRANCE. Disponible en: <https://www.legifrance.gouv.fr/affichJuriAdmin.do?oldAction=rechJuriAdmin\&idTexte=CET ATEXT000023109995\&fastReqld=759322029\&fastPos=1>. Accès en: 19 Sept. 2018.
} 
Gouvernement, les représentants des employeurs publics territoriaux et les représentants des employeurs publics hospitaliers. Ces négociations peuvent notamment porter sur lévolution des rémunérations, les conditions de travail, la santé des agents, la formation professionnelle continue ou encore l'égalité entre femmes et hommes ${ }^{12}$.

Pour les professionnels exerçant dans des établissements privés et ayant la qualité de salariés, la capacité de négociation des organisations syndicales peut s'exercer aussi bien au niveau national quau niveau d'une entreprise ou d'un établissement. Le pouvoir normatif des parties à la négociation collective s'est même particulièrement développé ces dernières années pour les entreprises du secteur privé au point que des accords négociés collectivement au niveau d'un établissement peuvent notamment imposer, sous certaines conditions, des évolutions de salaires ou de durée du travail à la hausse comme à la baisse ${ }^{13}$.

Mais si la négociation collective concernant les professionnels en établissement couvre un champ large au sujet des conditions de travail, d’emploi et de rémunération, elle ne vise pas des libertés professionnelles par définition déjà restreintes par une situation de subordination des professionnels vis-à-vis de la structure qui les emploie.

La force de la régulation des professions de santé par la négociation collective est plus marquante lorsquelle vise les professionnels exerçant leur activité sous une forme libérale.

La régulation de l'activité libérale des médecins, des sages-femmes, des chirurgiens-dentistes et des auxiliaires médicaux provient en partie de conventions nationales ou départementales négociées entre les organisations syndicales représentatives de la profession et les organismes de l'assurance maladie obligatoire. Ces conventions ont pour but d'organiser les relations entre la profession et lassurance maladie, notamment en déterminant les règles relatives à l'installation et aux tarifs. Les dispositions quelles contiennent sont de véritables règles contraignantes assorties de sanctions propres. L'instauration de ces conventions concernant les professionnels libéraux répondait très clairement à la volonté de préserver l'autonomie des professionnels libéraux face à la mise en place d'un système national de protection sociale accessible à tous et du secteur hospitalier. L'extension de leur domaine permet aujourd'hui d'affirmer que le pouvoir normatif des professions de santé provient en grande partie des conventions nationales ${ }^{14}$. Mais dans le même temps la norme conventionnelle tendrait à devenir de plus en plus le relais de la norme légale et

\footnotetext{
${ }^{12}$ Art. 8 bis de la loi ${ }^{\circ} 83-634$ du 13 juillet 1983, modifié par la loi n ${ }^{\circ} 2016-483$ du 20 avril 2016. LEGIFRANCE. Disponible en: <https://www.legifrance.gouv.fr/affichTexte.do?cidTexte=JORFTEXT000032433852\&categ orieLien=id>. Accès en: 19 Sept. 2018.

${ }^{13}$ Article L 2254-2 du Code du travail, modifié par la loi $n^{\circ} 2018-217$ du 29 mars 2018. LEGIFRANCE. Disponible en : < https://www.legifrance.gouv.fr/eli/loi/2018/3/29/MTRT1726748L/jo/texte>. Accès en : 19 Sept. 2018.

${ }^{14} \mathrm{HUTEAU}, \mathrm{G}$. Déclin ou renouveau des professions de santé dans l'élaboration de la norme de protection sociale. Droit Social, Paris, n. 2, p. 122, 2016.
} 
les possibilités de conclure des contrats en dehors des dispositifs conventionnels en relativiserait la force ${ }^{15}$. Les libertés des professionnels y conservent une place importante. Mais chaque convention d'exercice libéral doit en effet être approuvée par un arrêté ministériel pour entrer en vigueur et le contrôle de son application revient pour l'essentiel aux services de l'assurance maladie, qui gèrent un service public mais sont distincts de l'Etat.

Le caractère normatif des différents accords et conventions négociés collectivement ne fait aucun doute puisque ceux-ci instaurent des droits et devoirs qui peuvent en tant que tels faire lobjet d'une sanction. Le pouvoir régulateur ainsi reconnu à ces textes a d’ailleurs conduit le législateur à accroître les exigences à remplir par les organisations syndicales pour participer à une négociation collective et pour signer un texte valable juridiquement. Quel que soit le mode d'exercice de l'activité, seules les organisations syndicales représentatives peuvent négocier des dispositions conventionnelles, cette représentativité nécessitant notamment d’obtenir un score minimum au cours délections professionnelles ( $10 \%$ ou $8 \%$ selon les niveaux de négociation et les modes d'exercice). Lélection confère ainsi une légitimité plus grande aux organisations syndicales et plus conforme à l'importance normative des normes négociées. Les conditions de validité des accords conclus sont elles aussi assises sur ces scores électoraux.

Cette légitimité, parfois contestée par les professionnels, se rapproche de celle des ordres dont certaines instances, généralement départementales ou régionales, sont élues par les professionnels. Mais pour le reste, ordres et syndicats doivent être distingués. Les professionnels sont tenus de verser une cotisation auprès de leur ordre professionnel, mais ils sont libres d'adhérer ou non à un syndicat. Et si l'application de la norme conventionnelle aux professionnels ne nécessite pas que ceux-ci soient adhérents auprès du ou des syndicats signataires, cette norme ne concerne dans tous les cas qu'une partie de la profession, contrairement aux règles déontologiques. Concernant les professionnels libéraux, la norme conventionnelle peut même ne pas s'appliquer au professionnel qui fait une demande en ce sens.

La multiplicité des acteurs et des modes de régulation caractérise la régulation des professions de santé en France. La diversité des acteurs ainsi impliqués dans la régulation est telle qu'elle ne fait pas intervenir uniquement les ordres et les organisations syndicales. Les normes différentes des normes étatiques, conventionnelles et ordinales, telles que les recommandations de bonne pratique, sont ainsi de plus en plus nombreuses et instaurent une concurrence entre le pouvoir des experts scientifiques et celui de la communauté professionnelle ${ }^{16}$. Nous reviendrons très vite sur leur utilité.

\footnotetext{
${ }^{15}$ HUTEAU, G. op. cit.

${ }^{16}$ TABUTEAU, D. Référentiels, bonnes pratiques et recommandations: nouvelles normes ou "quasi normes" en santé? JDSAM Journal de Droit de La Sante et de L'assurance Maladie, n. 3, p. 16, 2015.
} 
Quoi qu'il en soit, la participation des représentants professionnels à lélaboration et à l'application des normes régissant l'activité des professionnels de santé nocculte jamais totalement l'intervention d'institutions étatiques, territoriales ou des services de l'assurance maladie. L'absence d' " autorégulation » s'explique par l'importance de la régulation professionnelle dans la conduite de la politique de santé et la réalisation de ses objectifs. Et cela passe déjà par une régulation des compétences professionnelles.

\section{La régulation des compétences professionnelles}

Une personne dont l'activité professionnelle est en lien avec la prévention des risques pour la santé, le traitement ou le suivi de la santé des personnes n'est pas nécessairement assimilée à un professionnel de santé en droit français. La loi française, codifiée au Code de la santé publique, énumère de manière limitative les professionnels de santé, ce qui en exclut par exemple les ostéopathes exerçant cette activité de manière exclusive ${ }^{17}$ et les chiropracteurs. Cette conception des professions de santé en apparence centrée sur la loi rejoint surtout une vision fermée des compétences professionnelles, souvent statique, parfois dynamique.

\section{L'accès à la profession}

Pour chaque profession de santé identifiée comme telle par la loi, le Code de la santé publique détermine le ou les diplômes qu'une personne doit avoir obtenu pour exercer la profession visée. Il faut toutefois préciser que l'Etat encadre aussi désormais la formation de certaines professions qui ne sont pas légalement des professions de santé mais qui se revendiquent comme telles et accomplissent des prestations proches de certains professionnels de santé ${ }^{18}$.

Le Code de la santé publique ou le Code de l'éducation fixent les modalités d’organisation et de réalisation des études nécessaires à l'obtention des diplômes. Ces modalités et les établissements pouvant dispenser les enseignements et délivrer les diplômes sont très divers. Depuis quelques années, le législateur français cherche toutefois à regrouper la prise en charge de certaines formations en santé, à unifier certains enseignements et à les attirer un peu plus dans le champ de compétences des collectivités territoriales et de l'université ${ }^{19}$. Après avoir partiellement transféré aux

\footnotetext{
${ }^{17} \mathrm{CE}, 23$ mars 2016, $\mathrm{n}^{\circ} 387595$ : " La pratique de l'ostéopathie ne constitue pas, au sens du code de la santé publique, l'exercice d'une profession paramédicale ". LEGIFRANCE. Disponible en: <https://www. legifrance.gouv.fr/affichJuriAdmin.do?idTexte=CETATEXT000032289626>. Accès en: 19 Sept. 2018.

${ }^{18}$ V. p. ex. Décret $n^{\circ} 2014-1505$ du 12 décembre 2014 relatif à la formation en ostéopathie. LEGIFRANCE. Disponible en: <https://www.legifrance.gouv.fr/eli/decret/2014/12/12/AFSH1427626D/jo>. Accès en: 19 Sept. 2018.

${ }^{19}$ Pour une synthèse des évolutions en cours et à venir, v. le communiqué de presse conjoint du Ministère des solidarités et de la santé et du Ministère de l'enseignement supérieur, de la recherche et de l'innovation du 14 mars 2018. FRANCE. Ministère des solidarités et de la santé. L'universitarisation des formations en santé. Disponible en: <https://solidarites-sante.gouv.fr/actualites/presse/dossiers-de-presse/article/Iuniversitarisation-des-formations-en-sante>. Accès en: 19 Sept. 2018.
} 
régions la responsabilité des formations paramédicales et sociales ${ }^{20}$, le législateur a ainsi créé une première année détudes supérieures commune aux études médicales, odontologiques, pharmaceutiques et de sage-femme ${ }^{21}$.

L' organisation du contenu des formations initiales, l'obligation d'obtenir un titre et la fixation des conditions d’obtention de ce titre pour exercer une activité professionnelle constituent les premiers éléments fondateurs d'une politique de santé publique cohérente et de la reconnaissance socio-juridique d'une profession. Il est possible d'indiquer que de récentes dispositions reconnaissant à des professionnels que ayant obtenu leur diplôme dans un autre Etat possibilité d'exercer partiellement les actes attachés à une profession ${ }^{22}$ remettent en cause cette fonction régulatrice essentielle de la formation et du titre qui s'y attache. Le caractère pénal de la sanction attachée à l'exercice illégal d'une profession de santé contribue à prendre conscience de l'importance du titre dans la régulation professionnelle, tout comme son objectif initial, toujours vivace, de protection de la sécurité des patients, auquel est venu s'adjoindre la recherche de prestations de qualité.

L' exercice de la profession dans un territoire, qu'il s'agisse ou non de la première installation, nécessite de sêtre inscrit au préalable auprès de lordre professionnel ou, pour les professions nétant pas rattachées à un ordre professionnel, d'avoir obtenu une autorisation d'exercice délivrée par le préfet représentant l'Etat dans la région d’exercice.

L' aptitude professionnelle et physique sont contrôlées à cette occasion, des représentants de la profession intervenant par l'intermédiaire de lordre ou d'une commission de professionnels consultée pour avis en l'absence d'ordre.

Mais les pratiques et les techniques sont évolutives et la régulation par les compétences nest pas seulement issue d'une formation initiale préalable à la délivrance d'un titre.

\section{L'obligation de faire évoluer les compétences}

Les professionnels doivent toujours veiller à donner des soins et exercer leur activité conformément aux données acquises de la science. Ils disposent pour cela de recommandations ou de référentiels à l'élaboration desquelles participent des acteurs du système de santé et de la communauté scientifique. Certains actes, procédés, techniques et méthodes peuvent être soumis à des règles de bonne pratique fixées par le Ministère de la santé sur avis de la Haute Autorité de Santé (HAS) ${ }^{23}$. La

\footnotetext{
${ }^{20}$ Art. L 4383-1 et s. Du CSP.

${ }^{21}$ Art. L 631-1 et s. du Code de l'éducation. LEGIFRANCE. Disponible en: <https://www.legifrance.gouv.fr/ affichCode.do?cidTexte=LEGITEXT000006071191>. Accès en: 19 Sept. 2018.

${ }^{22} V$. not. art. L 4112-7 du CSP pour les médecins, chirurgiens-dentistes et sages-femmes.

${ }^{23}$ Art. L $1115-1$ du CSP.
} 
HAS est une autorité publique indépendante à caractère scientifique et est composée de différents acteurs du monde de la santé, professionnels ayant ou non la qualité de professionnels de santé, représentants d'associations notamment. Parmi d’autres activités, elle élabore des guides de bon usage des soins ou des recommandations de bonne pratique à destination des professionnels ${ }^{24}$, des référentiels de qualité des soins et des pratiques professionnelles pour les médecins exerçant en établissement de santée ${ }^{25}$, des règles de bonne pratique sur la délivrance au patient des informations relatives à son état de santé26et sur les modalités d'accès à ces informations ${ }^{27}$. Son action vise aussi des non-professionnels de santé puisquelle établit des recommandations de bonne pratique pour les ostéopathes et les chiropracteurs ${ }^{28}$. C'est enfin la HAS qui habilite les professionnels chargés d'évaluer les pratiques d’autres professionnels. Les guides, recommandations et référentiels quélabore la HAS visent aussi bien la sécurité sanitaire et la qualité des prestations que lobjectif de maitrise des dépenses de santé.

Ces normes sont parfois critiquées pour leur incomplétude car elles prennent avant tout en compte des études scientifiques ${ }^{29}$. Le terme de norme n'est malgré tout pas usurpé les concernant car elles peuvent emporter des conséquences juridiques. Le Conseil d'Etat a clairement mis en évidence le lien entre les recommandations de bonne pratique de la HAS et l'obligation déontologique d'assurer au patient des soins fondés sur les données acquises de la science et a de ce fait reconnu leur caractère réglementaire. Les professionnels de santé visés par ces recommandations peuvent ainsi demander au juge de contrôler leur conformité à la loi, dès lors quelles peuvent leur être opposées lorsque leurs pratiques professionnelles ne s'y conforment $\mathrm{pas}^{30}$.

Les référentiels et guides divers sont en outre utilisés par les juges pour s'assurer que les soins donnés sont appropriés, ce qui peut les conduire à retenir ou écarter la responsabilité disciplinaire, civile ou pénale d'un professionnel ${ }^{31}$. Mais ces recommandations reposant sur des données scientifiques mouvantes, la Cour de cassation a reconnu que le caractère approprié des soins pouvait être apprécié

\footnotetext{
${ }^{24}$ Art. L 161-37, $2^{\circ}$ du Code de la sécurité sociale (CSS). LEGIFRANCE. Disponible en: <https://www.legifrance. gouv.fr/affichCode.do?cidTexte=LEGITEXTO00006073189\&dateTexte=>. Accès en: 19 Sept. 2018.

${ }^{25}$ Art. 1414-3-3 du CSP.

${ }^{26}$ Art. L 1111-2 du CSP.

${ }^{27}$ Art. L $1111-9$ du CSP.

${ }^{28}$ Art. R 161-72, $4^{\circ}$ du CSS.

${ }^{29}$ GALLAIS, M. La médecine générale face aux normes. Journal du Droit de la Santé et de l'Assurance Maladie, n. 4, 2015, p. 17. Disponible en: <http://www.sfmg.org/data/generateur/generateur_fiche/1312/fichier_ gallais_mg_face_normes_jdsam_decembre_201597d2a.pdf>. Accès en : 22 Oct. 2018.

${ }^{30} \mathrm{CE} 27$ avril 2011, $\mathrm{n}^{\circ}$ 334396. LEGIFRANCE. Disponible en: <https://www.legifrance.gouv.fr/affichJuriAdmin. do?idTexte=CETATEXTO00023946448>. Accès en: 19 Sept. 2018.

${ }^{31}$ BACACHE, M. La norme en jurisprudence. Journal du Droit de la Santé et de l'Assurance Maladie, n. 4, p. 29, 2015. V. p. ex. Court de cassacion, civile, (Cass.) $1^{\text {ère }}$ civ. 14 oct. $2010, n^{\circ} 09-68471$ : prescription d'un médicament qui n'était plus reconnu depuis des années comme un médicament de référence. LEGIFRANCE. Disponible en: <https://www.legifrance.gouv.fr/affichJuriJudi.do?oldAction=rechJuriJudi\&id Texte=JURITEXT000022921653\&fastReqld=82345665\&fastPos=1>. Accès en: 19 Sept. 2018.
} 
notamment au regard des recommandations de la HAS mais aussi d'études scientifiques qui nétaient pas prises en compte dans les recommandations à l'époque des faits $^{32}$.

Pour intégrer l'évolution des compétences dans la régulation professionnelle, il est nécessaire de donner aux professionnels les moyens d'évoluer, ce qui passe par une formation continue tout au long de leur activité. Larticle L 4021-1 du Code de la santé publique consacre pour cette raison un développement professionnel continu (DPC) qui constitue à la fois un droit et une obligation pour tout professionnel de santé. Tous les trois ans, un professionnel de santé doit justifier qu'il suit une démarche de DPC « comportant des actions de formation continue, d'analyse, d'évaluation et d'amélioration de ses pratiques et de gestion des risques ». Au vu de cette définition, le parcours de formation continue engagé par les professionnels n’intègre pas uniquement l'évolution des connaissances scientifiques.

L' étendue des évolutions possibles justifie que l'Etat soit accompagné par des instances professionnelles dans le choix des orientations de formation continue. Certes l'Etat dispose, par arrêté ministériel, du pouvoir de définir les orientations pluriannuelles de DPC. Mais il doit pour cela prendre en compte aussi bien les orientations qui s'inscrivent dans la politique nationale de santé qu'il définit que les orientations choisies dans la convention d’exercice libéral et celles déterminées par des conseils nationaux professionnels ou des représentants de la profession ou de la spécialité $^{33}$. A l'heure actuelle, ces orientations portent sur des thèmes nombreux et variés tels que le dépistage du cancer, la contraception et la santé sexuelle, la qualité de vie au travail des professionnels de santé ou encore la coordination des équipes de soins primaires ${ }^{34}$.

L' institution des conseils nationaux professionnels peut sembler obscurcir un peu plus le paysage déjà chargé de la représentation des professionnels de santé. Mais elle montre surtout à mon sens que cette mosaïque de la représentation professionnelle nécessite parfois d'être fondue en une entité propice au partage des opinions, des savoirs techniques et empiriques. Ces conseils concernent en effet les professionnels de santé qu'ils soient libéraux, fonctionnaires ou salariés et il revient aux représentants professionnels eux-mêmes d’en déterminer la composition ${ }^{35}$. Un conseil national professionnel peut ainsi être composé de l'ordre professionnel, de syndicats, représentatifs ou non, ou d'associations professionnelles. Ces conseils

\footnotetext{
${ }^{32}$ Cass. $1^{\text {ère }}$ civ. 5 avril 2018, n 17-15620. LEGIFRANCE. Disponible en: <https://www.legifrance.gouv.fr/ affichJuriJudi.do?oldAction=rechJuriJudi\&idTexte=JURITEXT000036803181>. Accès en : 19 Sept. 2018.

${ }^{33}$ Art. L 4021-2 du CSP.

${ }^{34}$ Arrêté du 23 avril 2018, JORF n du 28 avril 2018, texte $n^{\circ}$ 29. LEGIFRANCE. Disponible en: <https:// www.legifrance.gouv.fr/affichTexte.do;jsessionid=FCB26AF137C87A9AF52695403DAB1411.tplgfr23s_ 3 ?cidTexte $=$ JORFTEXTO00036847916\&dateTexte $=$ \&oldAction=rechJO\&categorieLien=id\&idJO=JORFCO NT000036847662>. Accès en: 19 Sept. 2018.

${ }^{35}$ Art. D $4021-3$ du CSP.
} 
alimentent les réflexions sur lévolution de leur profession et ont, plus précisément, pour mission de proposer des orientations et un parcours de $\mathrm{DPC}^{36}$. La réunion de différents représentants professionnels, aux modes d'exercice différents, peut faciliter lévolution d'une profession, dans ses pratiques et le contenu de sa régulation. Mais la cohésion entre la régulation des compétences et les pratiques professionnelles pose aussi la question des frontières entre professions.

\section{Persistances et remises en cause d'une conception statique des compétences}

Une conception dynamique de la régulation par les compétences professionnelles ne saurait être complète sans la possibilité pour les professionnels, spécialement les non-médecins, de dépasser l'encadrement statique et cloisonné de leurs missions. Seuls les médecins sont habilités à pratiquer tous les actes de diagnostic, de prévention et de traitement à condition que le domaine concerné n'excède pas leurs connaissances, leur expérience et les moyens dont ils disposent ${ }^{37}$. Les missions des autres professionnels de santé sont définies soit par des activités de manière circonscrite soit par une liste d'actes précisément identifiés. L'encadrement juridique des activités professionnelles de santé sest construit autour de la protection du monopole médical, essentiellement parce que les médecins étaient considérés comme les plus compétents professionnellement pour assurer les soins des patients dans les meilleures conditions de sécurité possibles. Les activités des autres professionnels de santé ont de ce fait été définies par un ensemble d’actes constituant autant de dérogations à ce monopole. Cela induit une régulation stricte des compétences des professionnels non médicaux, spécialement ceux qui sont désignés sous l’appellation d' « auxiliaires médicaux » par le Code de la santé publique. Or, des auxiliaires médicaux, notamment infirmiers, sont concrètement amenés à effectuer dans leur travail courant des actes qui légalement ressortent de la compétence d'un médecin ou d'un autre professionnel. En plus de présenter le risque juridique d'une sanction pour exercice illégal de la médecine, la non-reconnaissance juridique des compétences concrètes mises en œuvre par ces professionnels constitue un frein à leur évolution professionnelle et empêche les médecins de se décharger officiellement de tâches que d'autres professionnels peuvent tout à fait accomplir.

Le chemin est encore long pour faire évoluer la régulation professionnelle sur ce plan mais la loi du 26 janvier 2016 de modernisation du système de santés ${ }^{38} \mathrm{a}$ déjà franchi un pas en créant pour les auxiliaires médicaux la possibilité d'exercer « en pratique avancée ». L'exercice en pratique avancée désigne la mise en pratique

\footnotetext{
${ }^{36}$ Art. D $4021-2$ et R 4021-4 du CSP.

${ }^{37}$ Art. R $4127-70$ du CSP.

${ }^{38}$ Loi $n^{\circ}$ 2016-41, JORF du 27 janv. 2016. LEGIFRANCE. Disponible en: <https://www.legifrance.gouv.fr/ affichTexte.do?cidTexte=JORFTEXT000031912641\&categorieLien=id>. Accès en: 19 Sept. 2018.
} 
par un professionnel de santé de compétences professionnelles dépassant celles initialement attachées à sa profession et l'amenant notamment à disposer d'une expertise, d'une capacité de prise de décision dans des situations complexes et à réaliser des activités cliniques dans un domaine spécifique.

Le législateur a posé les bases d'un régime propre à ce type d'exercice professionnel en prévoyant d'abord qu'il pourra se faire en équipe coordonnée par un médecin, ou en assistance d'un médecin spécialiste ${ }^{39}$.

L'exercice en pratique avancée pourra comprendre « des activités d'orientation, d'éducation, de prévention ou de dépistage, des actes d'évaluation et de conclusion clinique, des prescriptions de produits de santé non soumis à prescription médicale ${ }^{40}$ ». Cela concernera les professionnels qui exercent leur activité depuis une durée minimale qui devra être fixée par décret et qui auront obtenu un diplôme universitaire spécifique. Deux décrets du 18 juillet 2018 ont mis en œuvre ces dispositions mais ils ne concernent que la profession infirmière ${ }^{41}$.

Officialiser juridiquement le franchissement des frontières de compétences posées par le Code de la santé publique reste une opération socialement et politiquement difficile. La régulation juridique intègre une certaine conception dynamique des compétences mais qui reste insuffisante au regard de l'utilité qu'un travail évolutif et collectif peut présenter pour une prise en charge plus globale et plus efficace des patients.

Ce survol de l'encadrement normatif des activités professionnelles sous l'angle des compétences confirme que dans le domaine de la santé la régulation professionnelle est fortement pluraliste et nous rapproche de l'idée de gouvernance compte tenu de la multitude d'organes de représentation professionnelle y intervenant. L'Etat conserve toutefois un pouvoir décisionnaire important et tend depuis quelques années, par l'intermédiaire de la loi, à rapprocher les politiques de formation. Les résistances socio-juridiques aux évolutions restent cependant fortes. Comme l'a dit Tabuteau, " on ne remet pas en cause sans heurt ni courage deux siècles de délégation de l'organisation sanitaire à un corps professionnel ${ }^{42}{ }^{2}$, le corps médical en l'occurrence. Et comme l'a montrée la mise en place du système de protection sociale, en France notamment, la régulation ne provient pas de l'Etat seul mais est le fruit de coalitions sociales et politiques ${ }^{43}$. L'observation vaut également pour les compétences professionnelles que l'Etat ne peut décider seul de faire

\footnotetext{
${ }^{39}$ Art. L 4301-1 du CSP.

${ }^{40}$ Art. L 4301-1, $1^{\circ}$ du CSP

${ }^{41}$ Décrets $n^{\circ} 2018-629$ et 2018-633, JORF du 19 juill. 2018.

${ }^{42}$ TABUTEAU, D. Démocratie sanitaire: les nouveaux défis de la politique de santé. Paris: Odile Jacob, 2013. p. 45.

${ }^{43}$ POLLET, G. La régulation au confluent des coalitions sociales et politiques. In: COMMAILLI, Jacques; JOBERT, Bruno (Dir). Les métamorphoses de la régulation politique. Paris: LGDJ, 1998. p. 329 et s. (coll. Droit et société).
} 
évoluer mais dont il doit assurer au mieux l'inscription dans le système de santé. Le fonctionnement d'une démocratie sociale digne de ce nom nécessite de lever de nombreuses ambiguités dont la régulation des professionnels de santé est en grande partie révélatrice, particulièrement lorsquelle touche aux libertés des professionnels.

\section{La régulation des libertés professionnelles}

Les inégalités dans l'accès à la santé et les renoncements aux soins sont dus à plusieurs facteurs, parmi lesquels peuvent notamment être identifiés des délais d'attente trop longs, une offre de prestations insuffisante dans certaines zones géographiques et un coût trop élevé pour certaines personnes ${ }^{44}$. La réduction de ces difficultés d'accès aux soins peut cependant se heurter à une opposition persistante entre une régulation par l'Etat de l'offre de santé et des tarifs et la défense d'un modèle libéral individualisé d'exercice de l'activité professionnelle en santé.

\section{La régulation de l'offre de prestations de santé}

Les établissements de santé ne sont pas les seuls à connaître une régulation de leur installation mais parmi les professionnels, l'encadrement n’est pas unifié.

La première étape de la régulation de l’offre professionnelle se fait au début des études permettant l'accès au titre professionnel. Certaines formations ont recours à des quotas et d'autres à des numerus clausus. Les quotas définissent un nombre limité détudiants admis à suivre la formation initiale dès la première année (infirmiers, psychomotriciens, orthophonistes, orthoptistes et audioprothésistes). Le numerus clausus désigne quant à lui le nombre détudiants acceptés en deuxième année détudes et concerne les études médicales, la pharmacie, lodontologie et la maïeutique. Ces seuils tendent à augmenter depuis environ 10-15 ans pour pallier certains déficits professionnels ${ }^{45}$.

Si le principe de la régulation démographique par un accès limité aux études est élargi à lensemble ou presque des professions de santé, en revanche la liberté pour le professionnel de s'installer une fois diplômé reste très diversement encadrée.

Certaines professions sont soumises à une régulation territoriale restreignant leur liberté d'installation. C'est le cas par exemple des pharmaciens d'officine, dont l'installation doit prendre en compte les besoins de la population ${ }^{46}$.

\footnotetext{
${ }^{44} \mathrm{~V}$. I'étude conduite par la CAISSE NATIONALE D'ASSURANCE MALADIE. L'OBSERVATOIRE DES NON RECOURS AUX DROITS ET SERVICES (ODENORE). Agir contre le renoncement aux soins: diagnostic, solutions et déploiement. Mars 2017.

${ }^{45}$ BARLET, Muriel; MARBOT, Claire (Dir.). Portrait des professionnels de santé - édition 2016. p. 84-88. (Collection Panoramas de la Drees - Santé). Disponible en: <https://drees.solidarites-sante.gouv.fr/ etudes-et-statistiques/publications/panoramas-de-la-drees/article/portrait-des-professionnels-de-santeedition-2016>. Accès en : 22 Oct. 2018.

${ }^{46}$ Art. L 5125-3 du CSP.
} 
Pour les infirmiers et les sages-femmes exerçant en libéral, la liberté d'installation est partiellement limitée par la convention nationale qui empêche les professionnels d'adhérer à la convention s'ils choisissent d'exercer dans des zones identifiées par les parties à la convention comme "sur-dotées ». A côté de l'effet dissuasif d'un non-conventionnement qui peut se révéler très couteux pour le professionnel, les conventions prévoient également pour ces professions un dispositif incitatif sous forme d'aide financière pour les infirmiers et sages-femmes libéraux s'installant dans des zones "sous-dotées », voire " très sous-dotées ».

Cette régulation combinant coercition et incitation n'a pas encore cours en revanche pour les médecins libéraux et les déserts médicaux ont la vie dure. La liberté d'installation fait partie des principes de la médecine libérale contenus dans la charte de la médecine libérale adoptée en 1927 et se retrouve à nouveau au cœur des revendications actuelles des médecins libéraux, en réaction au refus d'une «étatisation » du système de santé qui se serait notamment matérialisée par la création des Agences régionales de santé (ARS) en $2009^{47}$. Les dispositifs incitatifs se succèdent et sont aujourd'hui répartis entre la $\operatorname{loi}^{48}$ et la convention nationale. Certains concernent des médecins exerçant en libéral, généralistes ou non. D’autres visent des médecins exerçant en établissement pour pratiquer une activité en ambulatoire dans le cadre de leurs obligations de service. Les zones géographiques concernées sont généralement des zones caractérisées par une offre de soins insuffisante ou des difficultés dans l'accès aux soins identifiées comme telles par l'ARS. De manière générale, l'ARS peut conclure des contrats avec les professionnels de santé pour faire évoluer les pratiques, améliorer la qualité et la coordination des soins ${ }^{49}$.

Selon que le dispositif incitatif mobilisé est prévu par la loi ou la convention nationale, le contrat sera conclu entre le professionnel intéressé et l'ARS ou l'assurance maladie. Dans tous les cas, l'outil contractuel est omniprésent, l'adhésion volontaire des professionnels étant recherchée avant tout.

Bien qu'organisatrice du dispositif de permanence des soins, qui repose pour l'essentiel sur le volontariat des professionnels, le pouvoir des ARS reste limité par le pouvoir dont disposent par ailleurs les services de l'assurance maladie dans la régulation des professions de santé libérales. L'un des principaux obstacles à une régulation cohérente et égalitaire de l'offre de prestations de santé tient en effet à la dualité de régimes entre d'un côté une régulation prévue par la loi et confiée aux Agences régionales de santé (ARS) et d'un autre côté une organisation définie par

\footnotetext{
${ }^{47}$ HASSENTEUFEL, P. La défense de la médecine libérale, p. 5. La Vie des Idees. Disponible en: <http://www. laviedesidees.fr/IMG/pdf/me_decinslibe_raux16062015.pdf>. Accès en : 22 Oct. 2018.

${ }^{48}$ Art. L $1435-4$ et s. du CSP.

${ }^{49}$ Art. L $1435-4$ et L $1435-4-1$ du CSP.
} 
la convention d'exercice libérale conclue entre les syndicats représentatifs et l'Union nationale des caisses d'assurance maladie (UNCAM) ${ }^{50}$.

Les ARS pourraient pourtant conduire des transformations essentielles du système de santé en promouvant le travail en équipes pluridisciplinaires, en redistribuant les économies réalisées par une nouvelle organisation au profit des professionnels de santé travaillant sous cette forme et en recherchant leur adhésion à des recommandations de bonne pratique portant sur des évolutions dans l'organisation des activités professionnelles ${ }^{51}$. Cela faciliterait en outre le développement de professions qui ne sont pas des professions de santé mais qui permettraient aux professionnels de santé, notamment aux médecins, de consacrer davantage de temps au cœur de leur activité (gestion de la circulation des informations par exemple, recueil des requêtes,...).

Certains dispositifs vont certes dans ce sens mais sont peut-être encore insuffisants pour inscrire pleinement la régulation des professions de santé au cœur des transformations du système de santé. La régulation des tarifs peut encourir des reproches similaires, malgré les progrès accomplis.

\section{La régulation tarifaire}

Le niveau des tarifs payés par les patients et du remboursement octroyé par l'assurance maladie conditionne un accès aux prestations de santé ouvert à l’ensemble de la population sans distinction de revenus. Elle conditionne en dautres termes l'existence d'un système d'assurance maladie et de santé fondés sur l'égalité et la solidarité, toutes deux indissociables. Les créateurs de la Sécurité sociale française en 1945 nont pas procédé à un choix net entre la volonté d’autonomie professionnelle des professionnels de santé, des médecins notamment, et l'impératif d'un accès aux soins universel propre à une logique de solidarité.

Pour les professionnels travaillant en établissement, la rémunération est fixée par des grilles de salaires, certes différentes selon la nature de létablissement et le statut du professionnel mais qui laissent peu de place à la liberté individuelle du professionnel. La conciliation entre la satisfaction des objectifs de la politique de santé et la liberté des professionnels de santé en matière de revenus pose davantage question pour les professionnels exerçant une activité libérale.

La liberté tarifaire a été remise en cause dès les années 1960 par l'instauration de tarifs opposables fixés dans des conventions départementales puis nationales

\footnotetext{
${ }^{50}$ TABUTEAU, D. Déserts médicaux: un révélateur des contradictions des politiques de santé. JDSAM Journal de Droit de La Sante et de L'assurance Maladie, n. 2, p. 47, 2015.

${ }^{51}$ BRÉCHAT, P. H.; LOPEZ A. Pour des agences régionales de santé dotées de plus de moyens de régulation. Les Tribunes de la santé, n. 55, fasc. 2, p. 80, 2017. 10.3917/seve.055.0075.
} 
et que les professionnels sont tenus de respecter. Le principe des tarifs opposables implique que

la prise en charge ou le remboursement par l'assurance maladie de tout acte ou prestation réalisé par un professionnel de santé [...] est subordonné à leur inscription sur une liste établie [...] par l'Union nationale des caisses d'assurance maladie, après avis de l'Union nationale des organismes d'assurance maladie complémentaire et après avis, le cas échéant, de la Haute Autorité de santé lorsque la décision porte sur lévaluation du service attendu ou du service rendu d'un acte ou d'une prestation ${ }^{52}$.

Cette liste comprend des actes qui font l'objet d'une cotation déterminant le niveau de remboursement des actes et est composée d'une partie relative aux actes médicaux, la classification commune des actes médicaux (CCAM), et d'une partie relative aux actes des chirurgiens-dentistes, des sages-femmes et des auxiliaires médicaux, la nomenclature générale des actes professionnels (NGAP) . La facturation par un professionnel à l'assurance maladie de prestations non répertoriées par la nomenclature des actes constitue une violation de la convention pouvant donner lieu à un remboursement de l'indu et à une sanction disciplinaire.

Les conventions d'exercice professionnel prévoient en outre, pour les professionnels concernés par ces conventions, des références identifiant des soins et prescriptions inutiles ou dangereux et dont le non-respect peut entraîner le versement d'une contribution financière par le professionnel. Ces références sont établies par la HAS et peuvent ainsi constituer un dispositif de contrôle supplémentaire de l'activité des professionnels. Ceci étant, il revient à la convention elle-même de préciser dans quelles conditions ces références sont opposables aux professionnels et le montant de la contribution à verser le cas échéant.

L' éventuelle décision de sanction appartient dans tous les cas à la caisse d'assurance maladie, même si des représentants professionnels sont associés à l'instruction du dossier.

Il existe toutefois plusieurs possibilités pour les professionnels de dépasser les tarifs opposables, notamment, concernant les médecins, si un patient consulte un médecin spécialiste sans avoir consulté au préalable son médecin traitant ou pour les médecins titulaires de certains titres. Dans tous les cas, le médecin, même autorisé à pratiquer des dépassements, ne peut demander au patient des honoraires qui excèdent "le tact et la mesure» ${ }^{53}$, notion floue qui peut désigner des dépassements

\footnotetext{
${ }^{52}$ Art. L 162-1-7 du CSS.

${ }^{53}$ Art R 4127-53 du CSP et art. L 162-1-14-1, $2^{\circ}$ du CSS
} 
systématiques ${ }^{54}$, répétés malgré les mises en garde ${ }^{55}$, excessifs par rapport aux tarifs opposables ${ }^{56}$. Un devoir d'économie s'impose également aux professionnels de santé.

Les dépassements d'honoraires ne sont pas responsables de tous les maux du système de santé mais le tarif opposable est un si ce nest le pilier de l'assurance maladie car il est essentiel à légalité d’accès aux soins et à la prévention ${ }^{57}$.

Au-delà d'une liberté tarifaire conservée par certains professionnels, ce sont les principes du paiement à l'acte et du paiement direct qui peuvent faire obstacle à une prise en charge coordonnée et accessible à tous. Certes des mécanismes permettent aujourd'hui de dépasser le principe du paiement à l'acte. Une rémunération au forfait a été instaurée pour valoriser financièrement les médecins libéraux suivant certains types de patients et pathologies (affections de longue durée, patients âgés de plus de 80 ans,...). La rémunération à l'acte peut également être complétée par une rémunération conditionnée à l'atteinte d’objectifs de santé publique fixés par la convention d'exercice professionnel (suivi de pathologies chroniques, actes de prévention, efficience des prescriptions,...). Mais ces mécanismes restent insuffisants non seulement parce qu'ils sont facultatifs mais aussi parce qu'ils reposent sur l'atteinte d'indicateurs chiffrés qui ne peuvent embrasser toute l'activité des professionnels ${ }^{58}$. Ces limites persistent bien que la convention médicale signée en août 2016 ait intégré de nouveaux indicateurs.

Si l'adhésion à ces formes de rémunération reste toujours une option levée individuellement pour les professionnels libéraux, leur entrée dans le dispositiflimite inévitablement leur liberté professionnelle, l’assurance maladie vérifiant l'atteinte ou non des objectifs pour déterminer le droit à rémunération supplémentaire ${ }^{59}$. Mais une liberté tarifaire généralisée dans le cadre de l’assurance maladie obligatoire risquerait détendre un peu plus encore le rôle des assurances complémentaires dans la prise en charge des prestations. Les mécanismes de régulation des dépenses et le contrôle des professionnels pourraient s'en trouver accentués ${ }^{60}$.

\footnotetext{
${ }^{54} \mathrm{CE}, 9$ déc. 1994, $\mathrm{n}{ }^{\circ}$ 114650. LEGIFRANCE. Disponible en: <https://www.legifrance.gouv.fr/affichJuriAdmin. do? idTexte=CETATEXTO00007864563>. Accès en: 19 Sept. 2018.

${ }^{55} \mathrm{CE}, 29$ juill. 1996, $\mathrm{n}^{\circ}$ 129484. LEGIFRANCE. Disponible en: <https://www.legifrance.gouv.fr/ affichJuriAdmin.do?idTexte=CETATEXTO00007880796>. Accès en: 19 Sept. 2018.

${ }^{56} \mathrm{CE}, 29$ juill. 2002, $\mathrm{n}^{\circ} 215321$ (honoraires supérieurs de 10 fois aux tarifs conventionnels). LEGIFRANCE. Disponible en: <https://www.legifrance.gouv.fr/affichJuriAdmin.do?idTexte=CETATEXT000008090255>. Accès en: 19 Sept. 2018; CE, 18 févr. 1977, $\mathrm{n}^{\circ} 00557$ (honoraires six fois supérieurs aux tarifs conventionnels). LEGIFRANCE. Disponible en: <https://www.legifrance.gouv.fr/affichJuriAdmin.do?oldAct ion=rechJuriAdmin\&idTexte=CETATEXT000007650380\&fastReqld=2071669487\&fastPos=1 $>$. Accès en: 19 Sept. 2018.

${ }^{57}$ TABUTEAU, D. La liberté tarifaire? Droit Social, Paris, n. 4, p.424, avr. 2013.

${ }^{58}$ MARIE, R. Les évolutions à l'ouvre en matière de rémunération des médecins libéraux. Droit Social, Paris, p. 517, Mai, 2012.

${ }^{59}$ MARIE, R. Rémunération sur objectifs de santé publique et subordination des médecins libéraux. Revue de Droit Sanitaire et Social, n. 4, p. 615, juil./aout. 2013.

${ }^{60}$ TABUTEAU, D. La liberté tarifaire? Droit Social, Paris, n. 4, p.424, avr. 2013.
} 
La régulation de l'activité des professionnels de santé repose sur des équilibres entre une intervention de l'Etat nécessaire à une politique unifiée guidée par l'objectif (l'idéal ?) d'une meilleur santé pour tous, un partage de pouvoirs normatifs, des compromis historiques qui survivent pour défendre certaines libertés professionnelles et une dichotomie entre le système de santé et l'assurance maladie. L'organisation sanitaire est toujours en quête d'une cohérence, même si des tentatives d'unification et d'évolutions vers une autre façon d'exercer le travail en santé sont à l'œuvre. Le pluralisme dans la régulation reste pourtant nécessaire pour que les professionnels de santé puissent apporter aux pouvoirs publics leur savoir et leur adhésion à la politique de santé. Il devrait même être poussé plus loin en associant davantage les représentants des patients et des citoyens en général, et plus largement encore les citoyens euxmêmes pour connaître leurs besoins de santé et choisir au mieux les régulations professionnelles permettant d'y répondre. C'est peut-être aussi de cette manière que la régulation ne perdra pas en chemin l'idéal qu'elle doit poursuivre et que celui-ci ne soit pas une chimère.

\section{Références}

BACACHE, M. La norme en jurisprudence. Journal du Droit de la Santé et de l'Assurance Maladie, n. 4, p. 29-35, 2015.

BAILLY, J. M. Les déontologies. Paris: Presses universitaires d’Aix-Marseille, 2001.

BARLET, Muriel; MARBOT, Claire (Dir.). Portrait des professionnels de santé - édition 2016. p. 84-88. (Collection Panoramas de la Drees - Santé). Disponible en: <https://drees. solidarites-sante.gouv.fr/etudes-et-statistiques/publications/panoramas-de-la-drees/article/ portrait-des-professionnels-de-sante-edition-2016>. Accès en : 22 Oct. 2018.

BEC, C. La sécurité sociale: une institution de la démocratie. Paris: Gallimard, 2014.

BRÉCHAT, P. H.; LOPEZ A. Pour des agences régionales de santé dotées de plus de moyens de régulation. Les Tribunes de la santé, n. 55, fasc. 2, 2017. 10.3917/seve.055.0075.

BRISSY, S. La régulation des professions de santé par les ordres professionnels en droit français et européen. Revista de Direito Sanitário, São Paulo, v. 17, n. 3, p. 77-106, fev. 2017. Disponible en: <http://www.revistas.usp.br/rdisan/article/view/127777/124774>. https://doi. org/10.11606/issn.2316-9044.v17i3p77-106.

CAISSE NATIONALE D'ASSURANCE MALADIE. L'OBSERVATOIRE DES NON RECOURS AUX DROITS ET SERVICES (ODENORE). Agir contre le renoncement aux soins: diagnostic, solutions et déploiement. Mars 2017.

COMMAILlE, J.; JOBERT, B. (Dir.). Les métamorphoses de la régulation politique. Paris: LGDJ, 1998. (coll. Droit et société). 
FRANCE. Ministère des solidarités et de la santé. L'universitarisation des formations en santé. Disponible en: <https://solidarites-sante.gouv.fr/actualites/presse/dossiers-de-presse/article/luniversitarisation-des-formations-en-sante>. Accès en: 19 Sept. 2018.

GALLAIS, M. La médecine générale face aux normes. Journal du Droit de la Santé et de l'Assurance Maladie, n. 4, 2015, p. 17-25. Disponible en: <http://www.sfmg.org/data/generateur/ generateur_fiche/1312/fichier_gallais_mg_face_normes_jdsam_decembre_201597d2a.pdf > . Accès en : 22 Oct. 2018.

HASSENTEUFEL, P. La défense de la médecine libérale. La Vie des Idees. Disponible en: $<$ http://www.laviedesidees.fr/IMG/pdf/me_decinslibe_raux16062015.pdf>. Accès en : 22 Oct. 2018 . Po., 1997.

Les médecins face à l'Etat: une comparaison européenne. Paris: Presses de Sciences

HUTEAU, G. Déclin ou renouveau des professions de santé dans l'élaboration de la norme de protection sociale. Droit Social, Paris, n. 2, p. 121-125, 2016.

MARIE, R. Les évolutions à l’ouvre en matière de rémunération des médecins libéraux. Droit Social, Paris, p. 517-523, Mai, 2012.

. Rémunération sur objectifs de santé publique et subordination des médecins libéraux. Revue de Droit Sanitaire et Social, n. 4, p. 615-622, juil./aout. 2013.

POLLET, G. La régulation au confluent des coalitions sociales et politiques. In: COMMAILLI, Jacques; JOBERT, Bruno (Dir). Les métamorphoses de la régulation politique. Paris: LGDJ, 1998. (coll. Droit et société).

TABUTEAU, D. Démocratie sanitaire: les nouveaux défis de la politique de santé. Paris: Odile Jacob, 2013.

Déserts médicaux: un révélateur des contradictions des politiques de santé. JDSAM Journal de Droit de La Sante et de L'assurance Maladie, n. 2, p. 46-49, 2015.

La liberté tarifaire? Droit Social, Paris, n. 4, p.424-426, avr. 2013.

. Lavenir de la médecine libérale et le spectre de Monsieur Bovary. Droit Social, Paris, n. 4, p. 383-392, 2009.

Référentiels, bonnes pratiques et recommandations: nouvelles normes ou «quasi normes» en santé? JDSAM Journal de Droit de La Sante et de L'assurance Maladie, n. 3, 2015.

Stéphane Brissy - Maître de conférences à l'Université de Nantes. Membre de l'Institut Droit et Santé de l'Université Paris Descartes; INSERM UMR S 1145. Nantes, France. 